\title{
Next-Science: A Novel Antimicrobial Agent that Inhibits Biofilm Development by Escherichia coli Clinical Isolates on Urinary Tract Catheters
}

Sabrina Z. Siddiqui' ${ }^{1}$, Casandra Kruczek ${ }^{2}$, Jane A. Colmer-Hamood ${ }^{2}$, Matthew Myntti ${ }^{3}$ and Abdul N. Hamood ${ }^{4 *}$

${ }^{1}$ School of Medicine, Texas Tech University Health Sciences Centre, Lubbock, TX, USA

${ }^{2}$ Department of Medical Education, Texas Tech University Health Sciences Centre, Lubbock, TX, USA

${ }^{3}$ Next Science, Jacksonville, FL, USA

${ }^{4}$ Department of Immunology and Molecular Microbiology, Texas Tech University Health Sciences Centre, Lubbock, TX, USA

\begin{abstract}
Catheter-associated urinary tract infections (CAUTI) constitute about $40 \%$ of health-care associated infections in the United States. It is estimated that about $15 \%$ to $25 \%$ of hospitalized patients receive a urethral catheter at some point during hospitalization predisposing them to the development of CAUTI. Pathogenic bacteria colonize the surface of the catheter and develop highly resistant structures termed biofilms which protect them from the effects of diverse antibiotics. Escherichia coli are among the main causative agents of CAUTI. Due to the emergence of antibiotic resistant strains, it is critical to develop new novel antimicrobial agents. We recently showed that a novel anti-biofilm agent, Next Science, inhibited biofilm development by wound pathogens. In this study, we tried to determine if treating urinary tract catheters (UTC) with NS prevents the development if $E$. coli biofilms.

Three types of UTCs were cut into small pieces that were treated with NS. Biofilm development by an E. coli laboratory strain and several $E$. coli clinical isolates on treated and untreated pieces was accomplished using the microtiter plate assay. Biofilms developed on inner and outer surfaces of the catheters were quantified by determining the number of microorganisms (colony forming units) on each piece and visualized using confocal laser scanning microscopy (CLSM).

In comparison with untreated catheters, all three types of NS-treated catheters prevented biofilm development by tested E. coli strains. In addition, CLSM demonstrated the presence of $E$. coli biofilms on the inner and outer surfaces of the untreated but not treated catheters. Our results suggest that NS is a novel antimicrobial treatment to prevent biofilm development by pathogenic E. coli strains on UTCs.
\end{abstract}

Keywords: Urinary tract infections; Biofilm; Antimicrobial agents; E. coli

\section{Introduction}

Catheter-associated urinary tract infection (CAUTI) constitutes about $40 \%$ of healthcare associated infections in the USA [1]. Placing more than 30 million bladder catheters annually results thousands of cases of CAUTI [2]. Another contributing factor for CAUTI is the increased use of indwelling catheters; most hospitalized patients are catheterized for 2-4 days [3]. During catheterization, bacteria enter the urinary tract either through the extraluminal or the intraluminal route [4]. The bacteria utilize the extraluminal route early during the insertion of the catheter [4]. Alternatively, they may utilize this route later as the perineal bacteria ascend along the outer surface of the catheter [5]. The intraluminal route is utilized either when contaminated urine refluxes into the bladder from the collection bag or when a break in the closed drainage system occurs [5]. Most of CAUTI are caused by a single bacterial species (monomicrobial) including Staphylococcus epidermidis, Enterococcus faecalis, E. coli, and Proteus mirabilis [4-6]. These bacteria initially colonize the urinary tract catheter.

After colonizing the surface of the urinary catheter, contaminating bacteria form biofilms. The formation of the biofilm is essential for the development of CAUTI [7]. Factors within the bladder accumulate on the surface of the catheter and facilitate biofilm formation. These factors, which include host proteins, organic molecules, and conditioning film of electrolytes are important in the initial bacterial attachment [8]. Under certain conditions, bacteria form biofilms on both biotic and abiotic surfaces $[6,8]$. During biofilm formation, proliferating bacteria secrete extracellular polymeric substance (EPS) materials which protect them from the effects of antibiotics as well as different host responses [9]. Due to the significant role of these biofilms in the development of CAUTI, numerous approaches were utilized to prevent their formation on either the inner or outer surface of the urinary catheter. These approaches included modification of the catheter surface with antimicrobials included coating, matrix loading, and immersion in an antimicrobial agent. Among the antimicrobials that produced variable results are: hydrogels, silver, triclosan, nitric oxide, antibiotics, and quorum-sensing inhibitors $[4,10]$. The development of additional antimicrobial agents to prevent biofilm development on urinary tract catheters is essential. We recently described Next Science (NS), a novel antimicrobial agent that destroys the biofilm and eliminates individual the bacteria within it. NS gel prevented wound infection by different wound pathogens [11]. In addition, NS-treated tympanostomy tubes inhibited biofilm development by middle ear pathogens [12]. In this study, we examined the effectiveness of NS in preventing biofilm development by E. coli strains on urinary catheters.

*Corresponding authors: Abdul Hamood, Department of Immunology and Molecular Microbiology, Texas Tech University Health Sciences Centre, 36014 th Street STOP 6591 Lubbock, TX, USA, Tel: 806 7434057; Fax: 806 7432334; E-mail: abdul.hamood@ttuhsc.edu

Received December 11, 2017; Accepted December 20, 2017; Published December 21, 2017

Citation: Siddiqui SZ, Kruczek C, Colmer-Hamood JA, Myntti M, Hamood AA (2017) Next-Science: A Novel Antimicrobial Agent that Inhibits Biofilm Development by Escherichia coli Clinical Isolates on Urinary Tract Catheters. J Med Microb Diagn 6: 266. doi:10.4172/2161-0703.1000266

Copyright: (c) 2017 Siddiqui S, et al.. This is an open-access article distributed under the terms of the Creative Commons Attribution License, which permits unrestricted use, distribution, and reproduction in any medium, provided the original author and source are credited. 


\section{Materials and Methods}

\section{Bacterial strains and growth media}

Bacterial strains utilized in this study included the E. coli strain MM294 [13] and four E. coli clinical isolates (CF77, CF266, CF347, and CF358) obtained from cystic fibrosis (CF) patients presented at the pediatric clinic at Texas Tech University Medical Centre. The isolates were obtained through a protocol approved by the Institutional Review Board at Texas Tech University Health Sciences Centre, Lubbock, Texas. For general growth, strains were grown overnight in Luria-Bertani (LB) broth. Biofilms were developed using tryptic soy broth (TSB). To examine the biofilms using confocal laser scanning microscopy, we utilized the E. coli strain MM294/ pMRP9-1. Plasmid pMRP9-1 carries the gene that codes for green fluorescence protein (GFP) [14]. To maintain plasmid pMRP9-1 in MM294, we grew the strain in the presence of carbenicillin at a concertation of $50 \mu \mathrm{g} / \mathrm{mL}$.

\section{Next-Science}

Next-Science is a proprietary agent (PCT/US2012/059263) (Next Science, Jacksonville, FL) that was designed to eliminate biofilms by destabilizing the EPS matrix through chelation of calcium and to kill bacterial pathogens by removing proteins from bacterial membranes leading to cell lysis [11]. Next Science (NS) was obtained in an aqueous solution at a stock concentration of $1.3 \mathrm{mg} / \mathrm{mL}$ and was further diluted in phosphate buffered saline (PBS).

\section{Biofilm development}

The microtiter plate culture assay was utilized to develop the biofilms as previously described $[15,16]$. Briefly, aliquots of overnight cultures of the tested strain in LB broth were harvested, washed in $1 \mathrm{~mL}$ PBS, and diluted in PBS to an $\mathrm{OD}_{600}$ of 0.02 . Biofilms were developed on three types of urinary catheters; polyvinyl chloride (PVC) intermittent catheters, silicone catheters, and silicone-coated catheters. Each catheter was cut into small pieces $(0.25 \mathrm{~cm})$ and the pieces were washed in PBS and used in the biofilm assay. Catheter pieces were placed into the wells of a sterile 24-well polystyrene microtiter plate (Costar; Corning, Tewksbury, MA) containing TSB. Each well, which contained $1 \mathrm{~mL}$ of TSB, was inoculated with about 300 colony forming units (CFU) of the tested strain.

To investigate the effectiveness of NS in inhibiting biofilm development on the catheter pieces, $1 \mathrm{~mL}$ of either sterile PBS (negative control) or NS solution $(50 \mu \mathrm{g} / \mathrm{mL})$ was added to each well. After 24 $\mathrm{h}$ of additional incubation at $37^{\circ} \mathrm{C}$, the biofilms were quantified and visualized. Each experiment was repeated at least three times to confirm the reproducibility of the results.

\section{Quantification of the biofilms}

Each catheter piece was placed into a $1.5 \mathrm{~mL}$ microcentrifuge tube containing $1 \mathrm{~mL}$ of TSB and vigorously vortexed to disrupt the biofilm. The bacterial suspension was then serially diluted 10 - fold in TSB and $10 \mu \mathrm{L}$ aliquots of each dilution were spotted onto LB agar plates. The plates were incubated at $37^{\circ} \mathrm{C}$ for $24 \mathrm{~h}$ and the numbers of microorganisms $(\mathrm{CFU})$ in each catheter piece was determined using the following formula: (CFU counted X dilution factor X100). Each experiment was repeated at least 3 times for reproducibility.

\section{Visualization of the biofilms}

At the end of the biofilm development, each catheter piece was gently rinsed in $1 \mathrm{~mL}$ of PBS and the biofilm was visualized by confocal laser scanning microscopy (CLSM) using an Olympus IX71 Fluoview 300 confocal laser scanning microscope (Olympus America). The catheter piece was longitudinally split to visualize the biofilm on the inner and outer surfaces. Three-dimensional biofilm image reconstructions were performed using NIS Elements 2.2 software (Nikon Instruments).

\section{Statistical analyses}

Analyses were done using GraphPad Prism 6.05 (GraphPad Software, San Diego, CA). Differences between pairs were assessed by unpaired two-tailed $\mathrm{t}$ tests.

\section{Results and Discussion}

To prove the concept, we utilized the E. coli strain MM294, which is a laboratory strain. However, unlike other E. coli laboratory strains, MM294 maintained its wild type phenotype and none of its genes was mutated to facilitate cloning or expression studies. Preliminary experiments confirmed that NS completely inhibited the planktonic growth of MM294 (data not shown). We utilized three types of urinary catheters; PVC, silicone, and silicone coated (Materials and Methods). Each catheter was cut into $0.25 \mathrm{~cm}$ pieces and biofilm development on each piece was conducted using the microtiter plate assay as described in Materials and Methods. As shown in Figure 1, MM294 formed a considerable biofilm on all three types of catheters. In addition, at a concentration of $50 \mu \mathrm{g} / \mathrm{mL}$, NS completely inhibited the development of MM294 biofilms (Figure 1). We recovered no CFU from any of the catheter pieces (Figure 1). To confirm the effectiveness of any antimicrobial agent on a catheter, analysis of the inner and outer surfaces of the catheter is essential. Therefore, we visualized the MM294 biofilm on both sides on the catheter using CLSM. We selected the silicone catheter for such analysis. We utilized MM294 strain containing plasmid pMRP9-1 which carries the gene that codes for green fluorescence protein (GFP). As shown in Figure 2, MM294/ pMRP9-1, formed a well-developed biofilm on both sides of the silicone catheter. However, treatment with NS inhibited the development of the biofilm on both sides of the catheter (Figure 2). These results suggest that NS is effective in preventing E. coli biofilms on the exterior and interior surfaces of the silicone catheter.

We next examined, using the microtiter plate assay, the effect of NS on biofilms formed by E. coli clinical isolates obtained from patients on the silicone catheter. While all five tested isolates formed considerable biofilms on both sides of the catheter, they failed to do that in the presence of NS (Figure 3).

Antibiotic resistant mutants among different bacterial pathogens are developing at an alarming rate and the pathogens that cause CAUTI are no exception. One of the pathogens that contributes significantly

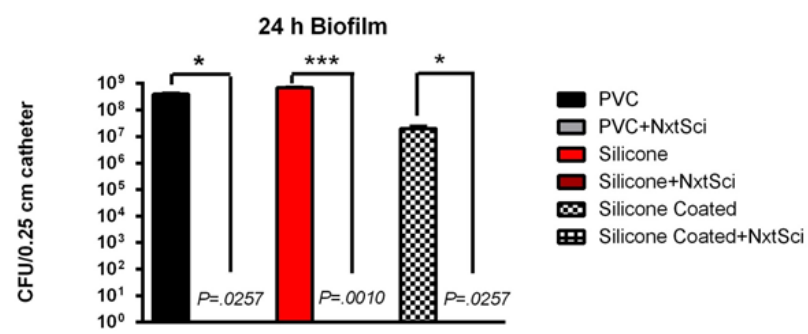

Figure 1: Next Science inhibits biofilm development by the E. coli strain MM294 on PVC, silicone, or silicone-coated urinary catheters. Biofilms were developed as described in the text and the number of microorganisms (CFU/0.25 cm piece of catheter) was determined. Values represent the average of three independent experiments, \pm standard error of the mean. 
Citation: Siddiqui SZ, Kruczek C, Colmer-Hamood JA, Myntti M, Hamood AA (2017) Next-Science: A Novel Antimicrobial Agent that Inhibits Biofilm Development by Escherichia coli Clinical Isolates on Urinary Tract Catheters. J Med Microb Diagn 6: 266. doi:10.4172/2161-0703.1000266

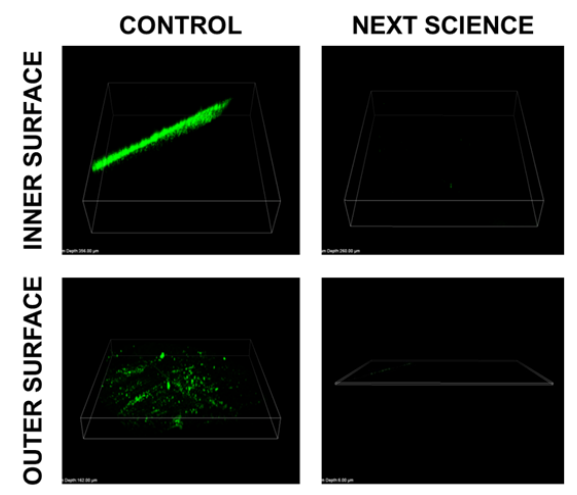

Figure 2: Next Science inhibits biofilm development by the E. coli strain MM 294 carrying the green fluorescent plasmid pMR9-1 on the outer and inne surface of the silicone coated catheter. Biofilms were developed as described in the text and visualized using confocal laser scanning microscopy.

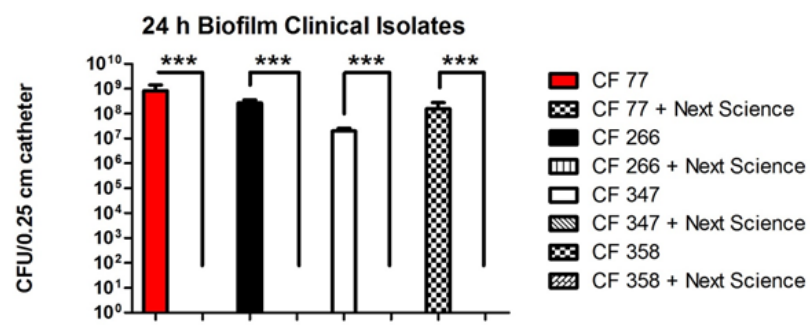

Figure 3: Next Science inhibits biofilm development by three E. coli clinical isolates on the surface of a silicone catheter. Biofilms were developed as described in the text and the number of microorganisms (CFU/ $0.25 \mathrm{~cm}$ piece of catheter) was determined. Values represent the average of three independent experiments, \pm standard error of the mean.

to CAUTI is E. coli $[4,5]$. In this study and using E. coli strains, we demonstrated the effectiveness of NS in: 1) preventing biofilm development on three different types of urinary catheters (Figure 1); 2) preventing biofilm development on both sides of a silicone catheter (Figure 2); and 3) preventing biofilm development by several clinical isolates on a silicone catheter (Figure 3). Thus, in clinical applications and within the urinary bladder of a catheterized patient, NS would potentially kill $E$. coli planktonic cells around the catheter and prevent those that survived from establishing a biofilm on either side of the catheter. We would also extend this analysis to include other pathogens (other than E. coli) obtained from patients with urinary tract infections. Another application of NS in to eliminate biofilms formed on urinary catheters.

\section{Conclusion}

An ideal approach in this regard is to utilize two agents; one that penetrates/destroys the EPS matrix (such as lysozyme) and another to kill the individual bacteria within the biofilm (such as antibiotics) $[17,18]$. However, as we previously demonstrated through in vitro and in vivo biofilm analyses NS is unique in that it contains both activities [11]. Therefore, in future experiments we would develop partial biofilms on both sides of the catheter and then treat the infected catheter with NS.

\section{Acknowledgements}

Funding for this study was supported in part by Next Science (Jacksonville, FL).

\section{References}

1. National Nosocomial Infections Surveillance (NNIS) (2004) System Report data summary from January 1992 through June 2004, issued October 2004 Am J Infect Control 32: 470-485.

2. Tambyah PA (2004) Catheter-associated urinary tract infections: diagnosis and prophylaxis. Int J Antimicrob Agents 24: S44-S48.

3. Weinstein JW, Mazon D, Pantelick E, Reagan-Cirincione P, Dembry LM, et al. (1999) A decade of prevalence surveys in a tertiary-care center: Trends in nosocomial infection rates, device utilization, and patient acuity. Infect Control Hosp Epidemiol 20: 543-548.

4. Siddiq DM, Darouiche RO (2012) New strategies to prevent catheter-associated urinary tract infections. Nat Rev Urol 9: 305-414.

5. Tambyah PA, Halvorson KT, Maki DG (1999) A prospective study of pathogenesis of catheter associated urinary tract infections. Mayo Clin Proc 74: 131-136.

6. Hentzer M, Teitzel GM, Balzer GJ, Heydornl A, Molin S, et al. (2001) Alginate overproduction affects $P$ seudomonas aeruginosa biofilm structure and function. J Bacteriol 183: 5395-5401.

7. Ong CL, Ulett GC, MabbettAN, Beatson SA, Webb RI, et al. (2008) Identification of type 3 fimbriae in uropathogenic $E$. coli reveals a role in biofilm formation. $J$ Bacteriol 190: 1054-1063.

8. Donlan RM, Costerton JW (2002) Biofilms: Survival mechanisms of clinically relevant microorganisms. Clin Microbiol Rev 15: 167-193.

9. Stewart PS, Costerton JW (2001) Antibiotic resistance of bacteria in biofilms Lancet 358:135-138.

10. Ha US, Cho YH (2006) Catheter-associated urinary tract infections: New aspects of novel urinary catheters. Int J Antimicrob Agents 28: 485-490.

11. Miller KG, Tran PL, Haley CL, Kruzek C, Colmer-Hamood JA, et al. (2014) Next Science wound gel technology, a novel agent that inhibits biofilm development by gram-positive and gram-negative wound pathogens. Antimicrob Agents Chemother 58: 3060-3072.

12. Banerjee D, Tran PL, Colmer-Hamood JA, Wang JC, Myntti M, et al. (2015) The antimicrobial agent, Next-Science, inhibits the development of Staphylococcus aureus and Pseudomonas aeruginosa biofilms on tympanostomy tubes. Int $\mathrm{J}$ Pediatr Otorhinolaryngol 79:1909-1914.

13. Meselson M, Yuan R (1968) DNA restriction enzyme from E. coli. Nature 217: $1110-1114$

14. Davies DG, Parsek MR, Pearson JP, Iglewski BH, Costerton JW, et al. (1998) The involvement of cell-to-cell signals in the development of a bacterial biofilm. Science 280: 295-298

15. Wang JC, Tran PL, Hanes R, Cordero J, Marchbanks J, et al. (2013) Inhibition of otopathogenic biofilms by organoselenium-coated tympanostomy tubes. JAMA Otolaryngol Head Neck Surg 13: 1009-1016.

16. Hammond A, Dertien J, Colmer-Hamood JA, Griswold JA, Hamood AN (2010) Serum inhibits $P$. aeruginosa biofilm formation on plastic surfaces and intravenous catheters. J Surg Res 159: 735-746.

17. Hou Y, Wang Z, Zhang P, Sun Y, Duan J, et al. (2017) Lysozyme associated liposomal gentamicin inhibits bacterial biofilm. Int J Mol Sci 18:784.

18. Thellin O, Zorzo W, Zorzo D, Delvenne P, Heinen E, et al. (2016) Lysozyme as a co-treatment during antibiotics use against vaginal infections: An in vitro study on Gardnerella vaginalis biofilm models. Int Microbiol 19: 101-107. 